\title{
A deep search for 21-cm absorption in high redshift damped Lyman- $\alpha$ systems
}

\author{
Nissim Kanekar ${ }^{1}$ and Jayaram N. Chengalur ${ }^{2}$ \\ 1 Kapteyn Institute, University of Groningen, Post Bag 800, 9700 AV Groningen, The Netherlands \\ 2 National Centre for Radio Astrophysics, Post Bag 3, Ganeshkhind, Pune 411 007, India \\ e-mail: chengalu@ncra.tifr.res.in
}

Received 18 September 2002 / Accepted 20 November 2002

\begin{abstract}
We present deep GMRT 21-cm absorption spectra of 10 damped Lyman- $\alpha$ systems (DLAs), of which 8 are at redshifts $z \gtrsim 1.3$. HI absorption was detected in only one DLA, the $z=0.5318$ absorber toward PKS $1629+12$. This absorber has been identified with a luminous spiral galaxy; the spin temperature limit $\left(T_{\mathrm{s}} \leq 310 \mathrm{~K}\right)$ derived from our observations continues the trend of DLAs associated with bright spirals having low spin temperatures. In seven of the remaining 9 systems, the observations place strong lower limits on the spin temperature of the HI gas.

We combine this sample with data taken from the literature to study the properties of all known DLAs with 21-cm absorption studies. The sample of DLAs which have been searched for $21-\mathrm{cm}$ absorption now consists of 31 systems, with $T_{\mathrm{s}}$ estimates available in 24 cases; of these, 16 are at $z<2$ and 8 at $z>2$, with 11 (all at $z<1$ ) having optical identifications. For the latter 11 DLAs, we find that all of the low $T_{\mathrm{s}}$ DLAs have been identified with large, luminous galaxies, while all the DLAs with high spin temperature $\left(T_{\mathrm{s}} \gtrsim 1000 \mathrm{~K}\right)$ have been identified either with LSBs or dwarfs. Further, we find no correlation between impact parameter and spin temperature; it is thus unlikely that the high measured $T_{\mathrm{s}}$ values for DLAs arise from lines of sight passing through the outskirts of large disk galaxies. Instead, the spin temperature of DLAs appears to correlate with the host galaxy type.

The trend (noted earlier by Chengalur \& Kanekar 2000) that low $z$ DLAs exhibit both high and low $T_{\mathrm{s}}$ values while high redshift $(z \gtrsim 3)$ DLAs only show high spin temperatures is present in this expanded data set. Based on this difference in spin temperatures, the Gehan test rules out the hypothesis that DLAs at $z>2$ and DLAs at $z<2$ are drawn from the same parent population at $\sim 99 \%$ confidence level.

Finally, we use the new GMRT spectra along with 2 spectra from the literature to estimate upper limits on the fraction of cold HI, $f_{\mathrm{CNM}}$, in DLAs at $z \gtrsim 3$. For local spirals, $f_{\mathrm{CNM}} \sim 0.5$; in contrast, we find that $f_{\mathrm{CNM}}<0.3$ in all 7 high $z$ absorbers, and $f_{\mathrm{CNM}}<0.1$ in 5 of the 7 cases.
\end{abstract}

Key words. galaxies: evolution: - galaxies: formation: - galaxies: ISM - cosmology: observations - radio lines: galaxies

\section{Introduction}

While molecular gas can be detected in emission at high redshifts (albeit only in a handful of somewhat unusual systems), it is as yet a severe challenge to detect neutral atomic gas in emission, even at redshifts as low as $z \sim 0.2$. As a result, absorption spectra against bright background sources provide the sole source of information on neutral atomic gas in the high redshift Universe. An important advantage of using absorption spectra as a probe of systems lying along the lines of sight to distant QSOs is that such studies tend to be unbiased, at least to first order - sources which give rise to absorption are presumably typical systems at that redshift. In contrast, emission studies of a flux-limited, high redshift sample are usually dominated by the brightest (often atypical) sources.

Send offprint requests to: Nissim Kanekar, e-mail: nissim@astro.rug.nl
The large absorption cross-section of the Lyman- $\alpha$ transition and the dominance of hydrogen in baryonic material makes the Lyman- $\alpha$ line one of the easiest to detect in QSO spectra. In fact, lines arising from neutral gas with column densities as low as $10^{13}$ atoms $\mathrm{cm}^{-2}$ can be routinely detected with present day telescopes. However, although these lines (the Lyman- $\alpha$ "forest") are by far the most numerous, they contain only a small fraction of the total neutral gas at high redshifts; most of the neutral gas is, in fact, contained in relatively rare, high column density $\left(N_{\mathrm{HI}} \gtrsim 10^{20}\right.$ atoms $\left.\mathrm{cm}^{-2}\right)$ systems, the so-called damped Lyman- $\alpha$ absorbers (DLAs). As the major repository of neutral gas at high redshift, these systems are natural candidates for the precursors of today's galaxies. However, despite their importance in the context of galaxy evolution, the nature of high redshift DLAs is presently quite controversial, with models ranging from large rapidly rotating disks (Wolfe et al. 1986; Prochaska \& Wolfe 1997) 
to merging sub-galactic blobs (Haehnelt et al. 1998) to outflows from dwarf galaxies (Schaye 2001) present in the recent literature.

A successful model for DLAs needs to not only reproduce the observed $\Omega_{\mathrm{g}}(z)$ and column density distribution but also to describe the observed physical conditions in the absorbing gas. The latter is important for our understanding of DLAs, quite independent of their precise size and structure. Conversely, knowledge of these conditions is useful in modelling the further temporal evolution of damped systems. HI 21-cm observations (possible only for those DLAs for which the background quasar is also radio loud) are particularly useful because they allow one to estimate the average spin temperature $T_{\mathrm{s}}$ of the absorbing gas. DLA spin temperatures have been, in general, found to be significantly higher than those typically seen in lines of sight through the Galactic disk, or in those through the disks of nearby galaxies (see e.g. Chengalur \& Kanekar 2000; Kanekar \& Chengalur 2001 and references therein). The only exceptions to the above are DLAs which have been identified as associated with low redshift $(z<0.6)$ spiral galaxies these systems all have low spin temperatures, comparable to those seen in the Milky Way and nearby spirals. Unfortunately, the number of systems for which deep 21-cm observations are available is relatively small; further, while the present data (Chengalur \& Kanekar 2000) suggest that high $z$ DLAs are different from low $z$ ones, the observations are currently biased toward systems at $z<1$, with not too many systems of the sample at high redshift. Are high redshift DLAs indeed systematically different from low redshift ones? Also, how significant is the fact that only DLAs associated with spiral galaxies have low spin temperatures? To find answers to these and similar questions, it is crucial to increase the number of systems with $21-\mathrm{cm}$ observations, particularly at high redshifts. We present, in this paper, deep Giant Meterwave Radio Telescope (GMRT) observations of 10 DLAs, 8 of which are at $z>1$. Two of these systems, the $z=2.9084$ DLA towards TXS 2342+342 and the $z=3.0619$ absorber towards PKS 0336-014 have been earlier observed by Carilli et al. (1996); the present observations are far more sensitive than the earlier ones. The observations themselves are summarised in Sect. 2, while the results are presented in Sect. 3 and their implications for physical conditions in DLAs discussed in Sect. 5.

\section{Observations and data analysis}

\subsection{The GMRT observations}

The GMRT has five currently operational frequency bands, at $150 \mathrm{MHz}, 233 \mathrm{MHz}, 327 \mathrm{MHz}, 610 \mathrm{MHz}$ and $1420 \mathrm{MHz}$. The present observations utilised the 327,610 and $1420 \mathrm{MHz}$ bands, which cover redshift ranges $z \sim 2.9-3.6, z \sim 1.15-$ 1.5 and $z \sim 0-0.6$ respectively, with reasonable sensitivity (i.e. better than half the sensitivity at the band centre). The observations were carried out between January and November 2001, using the 30-station FX correlator as the backend; this gives a fixed number of 128 channels over a bandwidth which can be varied between $64 \mathrm{kHz}$ and $16 \mathrm{MHz}$. All our observations (except for those of PKS 2128-123 (bandwidth = $2 \mathrm{MHz}$ ) and PKS 1629+12, which are described below) used a bandwidth of $1 \mathrm{MHz}$, sub-divided into 128 channels; this gave a channel resolution of $7.8 \mathrm{kHz}$. The number of available antennas varied between 18 and 26, due to various debugging and maintenance activities. One (or more) of the standard calibrators $3 \mathrm{C} 48,3 \mathrm{C} 147$ or $3 \mathrm{C} 286$ was used in all cases to calibrate the absolute flux scale and the system bandpass; these sources were observed at least every two hours in all cases, to ensure a good bandpass calibration. A nearby standard phase calibrator (see Cols. 4 and 5 of Table 1) was observed every forty minutes in all cases to determine the instrumental phase, except in the case of PKS 2128-123 which is itself a phase calibrator for the GMRT. Our experience with the GMRT indicates that the flux calibration is reliable to $\sim 15 \%$, in this observing mode. Observational details are summarised in Table 1, where the sources are arranged in order of increasing redshift; note that the values listed here for spectral resolution and rms noise are before any smoothing.

The only source towards which we detected 21-cm absorption, viz. PKS $1629+12$, was initially observed with a $1 \mathrm{MHz}$ bandwidth on the 12th and 22nd of October 2001, with 20 antennas and a total on-source time of $\sim 6$ hours. These observations resulted in the detection of a narrow 21-cm absorption feature; the source was hence re-observed on the 17th and 18th of November, 2001 with a $0.25 \mathrm{MHz}$ bandwidth (yielding a resolution of $1.95 \mathrm{kHz}$, i.e. $0.6 \mathrm{~km} \mathrm{~s}^{-1}$ ), to try to resolve out the absorption line. The total on-source time of these latter observations was 13 hours, with 25 antennas. In all cases, 3C286 was used for absolute flux and system bandpass calibration while the compact source $1640+123$ was used for phase calibration.

\subsection{Data analysis}

The data were analysed in classic AIPS using standard procedures. After the initial flagging and gain and bandpass calibration, continuum images were made for all sources and then used to self-calibrate the $U-V$ data. This was carried out in an iterative manner until the quality of the image was found to not improve on further self-calibration. In general, a few rounds of phase self-calibration were carried out on all fields, followed by one or two rounds of amplitude self-calibration. The continuum emission was then subtracted from the multi-channel $U-V$ data set, using the AIPS task UVSUB; any residual continuum was subtracted by fitting a linear baseline to the $U-V$ visibilities, using the task UVLIN. The continuum subtracted data were then mapped in all channels and a spectrum extracted at the quasar location from the resulting three dimensional data cube. In the case of PKS 1629+12, which showed 21-cm absorption, spectra were also extracted from other locations in the cube to ensure that the data were not corrupted by RFI. In general, all the observing frequencies were found to be reasonably free of RFI, except for sporadic interference on individual antennas which was edited out. In the case of multi-epoch observations of a single source, the spectra were shifted to the heliocentric frame outside AIPS and then averaged together. 
Table 1. Observing details.

\begin{tabular}{cccccccccc}
\hline \hline QSO & $z_{\text {abs }}$ & $\begin{array}{c}v_{\text {obs }} \\
\text { MHz }\end{array}$ & $\begin{array}{c}\text { Phase } \\
\text { Cal. }\end{array}$ & $\begin{array}{c}\text { Phase Cal. } \\
\text { Flux } \\
\text { Jy }\end{array}$ & $\begin{array}{c}\text { No. of } \\
\text { antennas }\end{array}$ & $\begin{array}{c}\text { Time } \\
\text { hrs }\end{array}$ & $\begin{array}{c}\text { Source } \\
\text { Flux } \\
\text { Jy }\end{array}$ & $\begin{array}{c}\text { Channel } \\
\text { resolution } \\
\mathrm{km} \mathrm{s}^{-1}\end{array}$ & $\begin{array}{c}\text { rms } \\
\text { mJy }\end{array}$ \\
\hline PKS 2128-123 & 0.4298 & 993.43 & $a$ & $a$ & 20 & 8 & 1.90 & 4.7 & 1.4 \\
PKS 1629+12 & 0.5318 & 927.28 & $1640+123$ & 2.5 & 25 & 13 & 2.35 & 0.6 & 2.8 \\
PKS 0215+015 & 1.3439 & 605.72 & $0204+152$ & 5.0 & 26 & 5 & 0.92 & 3.9 & 2.2 \\
QSO 0957+561A & 1.3911 & 594.04 & $0834+555$ & 7.4 & 26 & 6.5 & 0.59 & 3.9 & 2.4 \\
PKS 1354+258 & 1.4205 & 586.82 & 3 C286 & $b$ & 22 & 5.5 & $0.30^{c}$ & 4.0 & 2.8 \\
TXS 2342+342 & 2.9084 & 363.42 & 3 C19 & 8.3 & 23 & 8 & 0.31 & 6.4 & 2.8 \\
PKS 1354-107 & 2.966 & 358.15 & 3 C283 & 21 & 22 & 9.5 & 0.12 & 6.5 & 2.0 \\
PKS 0537-286 & 2.974 & 357.43 & $0521-207$ & 7.2 & 25 & 5 & 1.05 & 6.5 & 2.4 \\
PKS 0336-014 & 3.0619 & 349.69 & $0323+055$ & 7.4 & 26 & 5 & 0.94 & 6.7 & 2.1 \\
PKS 0335-122 & 3.178 & 339.97 & $0409-179$ & 6.1 & 18 & 5.5 & 0.68 & 6.9 & 1.9 \\
\hline
\end{tabular}

${ }^{a}$ No phase calibrator was used, as PKS $2128-123$ is itself a phase calibrator for the GMRT.

${ }^{b} 3 \mathrm{C} 286$ was also used as the flux calibrator; we hence do not have an independent estimate of its flux.

${ }^{c}$ PKS $1354+258$ is resolved by the GMRT synthesised beam; this is the peak flux.

\section{Results}

The final absorption spectra towards the ten sources of our sample are shown in Fig. 1, arranged in order of increasing redshift; the measured source fluxes, channel resolutions (in $\mathrm{km} \mathrm{s}^{-1}$ ) and rms noise values are listed in Cols. 8-10 of Table 1.

Our only detection of HI absorption is in the $z=0.5318$ DLA towards PKS 1629+12. Figure 2 shows a more detailed version of the GMRT $0.25 \mathrm{MHz} \mathrm{HI}$ spectrum towards this source; the spectrum has a resolution of $\sim 0.6 \mathrm{~km} \mathrm{~s}^{-1}$ and an rms noise of $2.8 \mathrm{mJy}$. Highly asymmetric $\mathrm{HI}$ absorption can be clearly seen, extending over a velocity width of $\sim 40 \mathrm{~km} \mathrm{~s}^{-1}$. The peak absorption occurs at a heliocentric frequency of 927.287 MHz, i.e. at a heliocentric redshift of $0.531787 \pm$ 0.000002 . This lies in between (and in reasonable agreement with) the redshifts of the damped Lyman- $\alpha$ line $\left(z_{\mathrm{DLA}}=0.532\right.$; Nestor et al. 2001) and the MgI absorption $\left(z_{\mathrm{MgI}}=0.5316\right.$ (Aldcroft et al. 1994; Barthel et al. 1990). (Note that Aldcroft et al. 1994 quote $z=0.5313$ for the strong MgII and FeII absorption lines.) The flux of PKS 1629+12 was measured to be $2.35 \mathrm{Jy}$; the peak optical depth is thus $\tau_{\max }=0.039$. The equivalent width of the profile is $\int \tau \mathrm{d} V=0.494 \pm 0.001 \mathrm{~km} \mathrm{~s}^{-1}$, obtained by integrating the observed absorption profile.

\section{The spin temperature}

In the case of an optically thin, homogenous cloud in thermal equilibrium, its HI column density, $N_{\mathrm{HI}}$, 21-cm optical depth, $\tau$, and spin temperature, $T_{\mathrm{s}}$, are related by the expression (e.g. Rohlfs 1986)

$N_{\mathrm{HI}}=\frac{1.823 \times 10^{18} T_{\mathrm{s}}}{f} \int \tau \mathrm{d} V$,

where $N_{\mathrm{HI}}$ is in $\mathrm{cm}^{-2}, T_{\mathrm{s}}$ in $\mathrm{K}$ and $\mathrm{d} V$ in $\mathrm{km} \mathrm{s}^{-1} . f$ is the covering factor of the absorber. Of course, a given line of sight through an absorber is quite likely to pass through a number of clouds, with different spin temperatures and column densities. In such a situation, the spin temperature obtained using the above expression is the column density weighted harmonic mean of the spin temperatures of the individual clouds (provided, of course, that all the clouds are optically thin).

It should be noted that Eq. (1) relates the HI column density to the integrated optical depth $\int \tau \mathrm{d} V$; this means that, in the case of a non-detection, one needs to know the shape of the absorption profile to compute a lower limit on the spin temperature. This shape is, of course, not a priori known. However, since our limits on the optical depth imply that the HI line is optically thin, the line can be assumed to have a Gaussian shape; here, $\int \tau \mathrm{d} V=1.06 \tau_{\max } \Delta V$, where $\tau_{\max }$ is the peak optical depth (or the limit on the peak) and $\Delta V$ is the FWHM of the Gaussian. Further, for a gas cloud in thermal equilibrium, the kinetic temperature $T_{\mathrm{k}}$ (with $T_{\mathrm{s}} \approx T_{\mathrm{k}}$, for a single cloud) and the FWHM of the observed line are related $\left(T_{\mathrm{k}} \approx 21.855 \Delta V^{2}\right.$, with $T_{\mathrm{k}}$ in $\mathrm{K}$ and $\Delta V$ in $\mathrm{km} \mathrm{s}^{-1}$ ); care must hence be taken to derive the limits on $T_{\mathrm{s}}$ in a self-consistent manner, so that the velocity width over which the integral is carried out is consistent with (at least) thermal broadening of the gas at the derived temperature (any bulk kinematic motion will further broaden the absorption features; we will assume that no such motions are present). For example, if the $3 \sigma$ lower limit on the spin temperature is $10000 \mathrm{~K}$, one should use $\Delta V \gtrsim 20 \mathrm{~km} \mathrm{~s}^{-1}$, while, if the limit is $1000 \mathrm{~K}$, it is appropriate to use $\Delta V \gtrsim 7 \mathrm{~km} \mathrm{~s}^{-1}$. We have, in the following analysis, used $\Delta V \approx 7,15$ and $20 \mathrm{~km} \mathrm{~s}^{-1}$ for temperatures $T_{\mathrm{s}} \sim 1000,5000$ and $10000 \mathrm{~K}$, respectively, and have smoothed the spectra to the above resolutions while computing the spin temperature.

The HI column density of a DLA can be directly estimated from the equivalent width of the Lyman- $\alpha$ profile. The original criterion for a system to be classified as a damped absorber $\left(N_{\mathrm{HI}} \geq 2 \times 10^{20} \mathrm{~cm}^{-2}\right)$ was an observational one, linked to the ability to identify damped profiles in medium resolution spectra from the relatively small telescopes used to carry out the early DLA surveys. Throughout this paper, however, we will consider all systems for which the Lyman- $\alpha$ profile shows damping wings as DLAs, regardless of whether or not their 

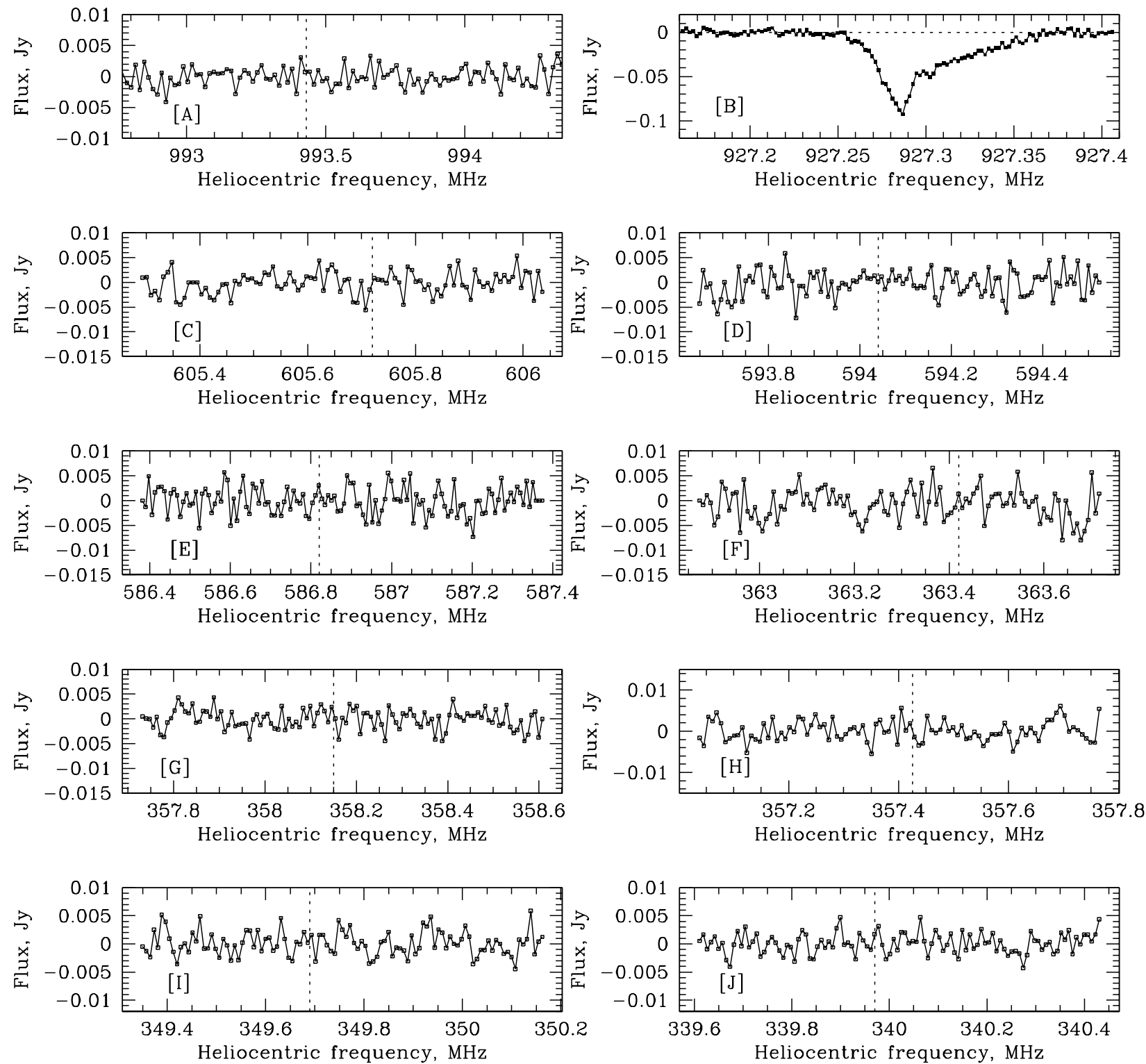

Fig. 1. GMRT HI absorption spectra towards the ten sources of our sample, arranged in order of increasing redshift. [A] $z=0.4298$ DLA towards PKS 2128-123. [B] $z=0.5318$ DLA towards PKS 1629+12. [C] $z=1.3449$ DLA towards PKS 0215+015. [D] $z=1.3911$ DLA towards QSO 0957+561A. [E] $z=1.4205$ DLA towards PKS 1354+258. [F] $z=2.9084$ DLA towards TXS 2342+342. [G] $z=2.966$ DLA towards PKS 1354-107. [H] $z=2.974$ DLA towards PKS 0537-286 [I] $z=3.0619$ DLA towards PKS 0336-014. [J] $z=3.178$ DLA towards PKS 0335-122.

HI column density is greater than the traditionally used threshold. We hence retain the absorbers towards PKS $0215+015$ and PKS 2128-123 in our DLA sample, despite their HI column densities being less than $10^{20} \mathrm{~cm}^{-2}$.

Given the HI column density, a search for $21-\mathrm{cm}$ absorption directly yields the spin temperature of the absorbing gas (or limits on the temperature, in the case of nondetections of absorption), provided the covering factor $f$ is known. Unfortunately, the radio continuum from quasars tends to arise from a far more extended region than the optical or UV emission and it may thus transpire that some fraction of the radio continuum may "leak out" from around the cloud and result in an incorrectly estimated spin temperature. Similarly, the line of sight along which the 21-cm optical depth has been measured need not be the same as that for which one has an estimate for the HI column density from the Lyman- $\alpha$ line. The fraction of radio emission originating in compact components spatially coincident with the UV point source can usually, however, be measured from VLBI observations (when such observations are available at sufficiently low frequencies). One can then estimate $f$ and hence, the spin temperature; this is done next, for the ten sources of this paper, in order of increasing right ascension. The results are summarised in Table 2 in which Cols. 4 to 8 contain, respectively, the velocity width $\Delta V$ used to compute $T_{\mathrm{s}}$, the spectral rms over this (or a slightly smaller) velocity width, the peak optical depth $\tau_{\max },(1 / f) \int \tau \mathrm{d} V$, and the spin temperature $T_{\mathrm{s}}$ (or limits on the last three quantities). 
Table 2. The spin temperature for the 10 DLAs observed here.

\begin{tabular}{ccccccccc}
\hline \hline QSO & $\begin{array}{c}z_{\mathrm{abs}} \\
\text { Q }\end{array}$ & $\begin{array}{c}N_{\mathrm{HI}} \times 10^{20} \\
\mathrm{~cm}^{-2}\end{array}$ & $\begin{array}{c}\Delta V^{a} \\
\mathrm{~km} \mathrm{~s}^{-1}\end{array}$ & $\begin{array}{c}\mathrm{rms}^{c} \\
\mathrm{mJy}\end{array}$ & $\begin{array}{c}\tau_{\max }^{d} \\
(1 / f) \int \tau \mathrm{d} V^{e} \\
\mathrm{~km} \mathrm{~s}^{-1}\end{array}$ & $\begin{array}{c}T_{\mathrm{s}} \\
\mathrm{K}\end{array}$ & Refs. $^{f}$ \\
\hline PKS 2128-123 & 0.4298 & $0.25 \pm 0.06$ & 7 & 1.2 & $<0.0019$ & $<0.014$ & $>980$ & 1 \\
PKS 1629+12 & 0.5318 & 2.8 & 0.6 & 2.8 & 0.039 & $0.494 \pm 0.001$ & 310 & 2 \\
PKS 0215+015 & 1.3439 & 0.8 & 7.8 & 1.6 & $<0.0052$ & $<0.043$ & $>1020$ & 3 \\
QSO 0957+561A & 1.3911 & $2.1 \pm 0.5$ & - & - & - & - & - & 4 \\
PKS 1354+258 & 1.4205 & $32 \pm 2$ & - & - & - & - & - & 4 \\
TXS 2342+342 & 2.9084 & $20 \pm 0.5$ & 15 & 2.0 & $<0.0192$ & $<0.306$ & $>3585$ & 5 \\
PKS 1354-107 & 2.966 & 6.0 & 6.5 & 2.0 & $<0.05$ & $<0.345$ & $>955$ & 6 \\
PKS 0537-286 & 2.974 & 2.0 & 10 & 1.9 & $<0.0054$ & $<0.058$ & $>1890$ & 6 \\
PKS 0336-014 & 3.0619 & $16 \pm 1.3$ & 20 & 1.4 & $<0.0045$ & $<0.095$ & $>9240$ & 7 \\
PKS 0335-122 & 3.178 & $6 \pm 1$ & 15 & 1.2 & $<0.0053$ & $<0.084$ & $>3920$ & 6 \\
\hline
\end{tabular}

${ }^{a} \Delta V$ is the FWHM used to compute the spin temperature in the case of non-detections (see the text for more details). For 1629+12, it is the velocity resolution of the observations.

${ }^{b}$ The large uncertainty in the covering factor makes it difficult to estimate the spin temperature (see the text for more details).

${ }^{c}$ This is the rms acually measured from the smoothed spectra. The spectra were smoothed to a velocity resolution given in the preceeding column, except for the following cases for which the rms was measured at a slightly better spectral resolution than $\Delta V$, viz $12.8 \mathrm{~km} \mathrm{~s}^{-1}$ for TXS 2342+342, $13.4 \mathrm{~km} \mathrm{~s}^{-1}$ for PKS 0336-014 and $13.8 \mathrm{~km} \mathrm{~s}^{-1}$ for PKS 0335-122.

${ }^{d}$ All limits (in this and other columns) correspond to $3 \sigma$.

${ }^{e}$ The covering factor $f$ has been set to unity in all cases (see the text for details).

${ }^{f}$ References for the HI column densities: 1. Ledoux et al. (2002); 2. Nestor et al. (2001); 3. Lanzetta et al. (1995); 4. Rao \& Turnshek (2000); 5. White et al. (1993); 6. Ellison et al. (2001a); 7. Prochaska et al. (2001).

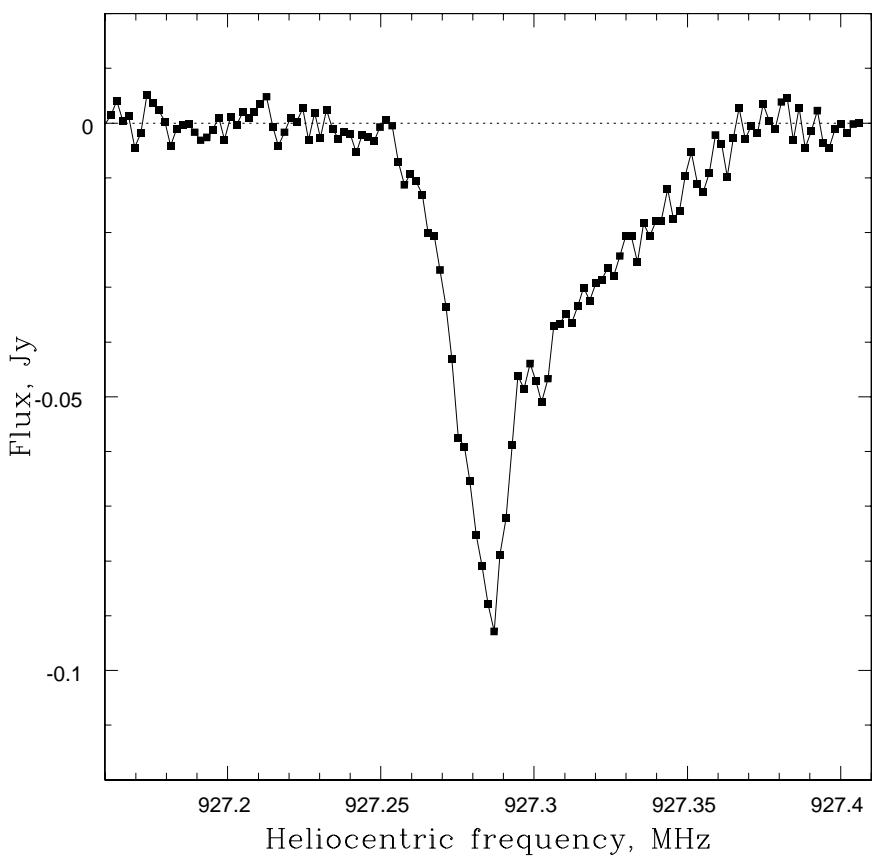

Fig. 2. High resolution $\left(0.6 \mathrm{~km} \mathrm{~s}^{-1}\right)$ GMRT HI absorption spectrum towards PKS $1629+12$. Asymmetric HI absorption is clearly visible, with the peak optical depth at $z=0.531787$.

1. VLBA maps of PKS $0215+015$ at 2.3 and $8.4 \mathrm{GHz}$ (from the Radio Reference Frame Image Database RRFID) show that the source is exceedingly compact, with most of the flux contained within the central 30 milli-arcseconds. The quasar also has a flat spectrum between $8.4 \mathrm{GHz}$ and $610 \mathrm{MHz}$, again implying that it is quite likely to be compact even at the lower frequencies. It is hence likely that the covering factor $f$ is close to unity. The HI column density of the $z=1.345$ DLA is $N_{\mathrm{HI}}=8 \times 10^{19} \mathrm{~cm}^{-2}$ (Lanzetta et al. 1995); the spin temperature of the absorber is then $T_{\mathrm{s}}(3 \sigma)>1020 \mathrm{~K}$ (using $\Delta V=7.8 \mathrm{~km} \mathrm{~s}^{-1}$ ).

2. The $z=3.178$ DLA towards PKS 0335-122 is the highest redshift system of our sample, with $N_{\mathrm{HI}}=6 \pm 1 \times$ $10^{20} \mathrm{~cm}^{-2}$ (Ellison et al. 2001a). Unfortunately, no VLBI information exists in the literature on the background quasar; however, this source has a fairly flat spectrum between $5 \mathrm{GHz}$ and $1.4 \mathrm{GHz}$, with fluxes of $380 \mathrm{mJy}$ and $420 \mathrm{mJy}$ at these two frequencies. This spectral flatness implies that the flux is likely to arise from a fairly compact source component, which is hence likely to be covered by the DLA. This gives a lower limit to the covering factor, $f \geq 0.7$, which yields $T_{\mathrm{s}}(3 \sigma)>2740 \mathrm{~K}$; if $f=1$, one has $T_{\mathrm{s}}(3 \sigma)>3920 \mathrm{~K}\left(\Delta V=15 \mathrm{~km} \mathrm{~s}^{-1}\right)$.

3 . In the case of PKS 0336-014, $5 \mathrm{GHz}$ VLBI observations (Gurvits et al. 1994) have shown that the source is highly compact at this frequency, with the entire flux contained within the central $\sim 7$ milli-arcseconds. However, the source also has a negative spectral index, indicating the presence of some extended emission. In the absence of VLBI information at lower frequencies, we will assume a covering factor of unity. The HI column density of the $z=3.0619$ DLA is $N_{\mathrm{HI}}=1.6 \pm 0.13 \times$ $10^{21} \mathrm{~cm}^{-2}$ (Prochaska et al. 2001) giving a spin temperature $T_{\mathrm{s}}(3 \sigma)>9240 \mathrm{~K}\left(\Delta V=20 \mathrm{~km} \mathrm{~s}^{-1}\right)$. Carilli et al. (1996) estimated $T_{\mathrm{s}}>2500 \mathrm{~K}$ for this DLA.

4. PKS 0537-286 has an exceedingly flat spectrum between $8.4 \mathrm{GHz}$ and $327 \mathrm{MHz}$; for example, the QSO flux density was measured to be 0.9 Jy at $8 \mathrm{GHz}$ (Parkes survey, Wright \& Otrupcek 1990), 1.2 Jy at $4.85 \mathrm{GHz}$ (Griffith et al. 1994), $838 \mathrm{mJy}$ at $1.4 \mathrm{GHz}$ (NRAO-VLA Sky Survey, Condon et al. 1998) and 1.05 Jy in our GMRT $327 \mathrm{MHz}$ image. This implies that the source is likely 
to be highly compact. VLBA observations at $5 \mathrm{GHz}$ (Fomalont et al. 2000) have shown that the flux within the central 10 milli-arcseconds is $\sim 500 \mathrm{mJy}$, implying a covering factor greater than 0.5 . In fact, these observations recovered the entire $5 \mathrm{GHz}$ flux of $1.2 \mathrm{Jy}$ on the shortest VLBA baseline $\left(2 \times 10^{6}\right.$ wavelengths, Hirabayashi et al. 2000), implying that the entire source is contained within $\sim 0.1$ arcsec. It is thus quite likely that the covering factor is close to unity; we will use $f=1$ in this analysis. The HI column density of the $z=2.9742$ DLA has been found to be $2.0 \times$ $10^{20} \mathrm{~cm}^{-2}$ (Ellison et al. 2001a); we then obtain a $3 \sigma$ lower limit of $1890 \mathrm{~K}$ on the spin temperature of the absorbing gas $\left(\Delta V=10 \mathrm{~km} \mathrm{~s}^{-1}\right)$.

5. VLBI mapping of QSO 0957+561 has been carried out on a number of occasions, at various frequencies at and above $1.6 \mathrm{GHz}$ (see, for example, Garrett et al. 1994, Campbell et al. 1995). The flux of component A has been measured to be only about $32 \mathrm{mJy}$ at $1.6 \mathrm{GHz}$ (Garrett et al. 1994); clearly, most of the flux seen in the $610 \mathrm{MHz}$ GMRT map comes from extended emission which is unlikely to be covered by the DLA. The covering factor is thus quite uncertain for this absorber, although it is likely to be low. Given this, limits on the spin temperature derived from the present data are unlikely to be reliable. In fact, it is known that the DLA does not cover the second image (B) of this gravitational lens system (Rao \& Turnshek 2000). We will hence not include this system in the later analysis and also do not list limits on the spin temperature and the optical depth in Table 2.

6. PKS $1354+258$ is also an extended source at $610 \mathrm{MHz}$, with no VLBI information available in the literature; the DLA covering factor is hence again quite uncertain. However, the extremely high column density of the absorber $\left(N_{\mathrm{HI}}=3.2 \pm 0.2 \times\right.$ $10^{21} \mathrm{~cm}^{-2}$; Rao \& Turnshek 2000) results in high lower limits on the spin temperature even in the case of low covering factors. For example, $f=0.1$ gives $T_{\mathrm{s}}(3 \sigma)>1100 \mathrm{~K}$, while $f=1$ would imply $T_{\mathrm{s}}(3 \sigma)>7000 \mathrm{~K}$ (assuming velocity widths of $8 \mathrm{~km} \mathrm{~s}^{-1}$ and $20 \mathrm{~km} \mathrm{~s}^{-1}$ respectively). We will however again not quote a spin temperature in Table 2 due to the uncertainties in the covering factor.

7. No information exists in the literature regarding the VLBI structure of PKS 1354-107. However, the source has a strongly inverted spectrum and is thus likely to be very compact. We will hence assume a covering factor of unity. Combining this with the HI column density of $6 \times$ $10^{20} \mathrm{~cm}^{-2}$ (Ellison et al. 2001a) gives $T_{\mathrm{s}}(3 \sigma)>955 \mathrm{~K}(\Delta V=$ $\left.6.5 \mathrm{~km} \mathrm{~s}^{-1}\right)$.

8. The HI column density of the $z=0.5318$ DLA towards PKS $1629+12$ is $N_{\mathrm{HI}}=2.8 \times 10^{20} \mathrm{~cm}^{-2}$ (Nestor et al. 2001). PKS $1629+12$ is a well-known compact steep spectrum source with an extremely one-sided structure (Saikia et al. 1990). The 2.3 GHz VLBI map of Dallacasa et al. (1998) shows two components with fluxes of $110 \mathrm{mJy}$ and $150 \mathrm{mJy}$. Only the $110 \mathrm{mJy}$ western component is seen in their $8.4 \mathrm{GHz}$ map, with almost the same flux; they hence identify this as the flat spectrum core of the source. The separation between the two components is $1.14 \operatorname{arcsec}$ (i.e. $\sim 6.4 \mathrm{kpc}$ at $z=0.5318$, using $H_{0}=65 \mathrm{~km} \mathrm{~s}^{-1} \mathrm{Mpc}^{-1}$ and an $\Omega=1, \Lambda=0$ cosmology). On the other hand, the core flux density was measured to be
$179 \mathrm{mJy}$ at $408 \mathrm{MHz}$ from a $\sim 0.6 \times 0.5$ arcsec resolution Merlin image (Saikia et al. 1990). Using the $2.3 \mathrm{GHz}$ and $408 \mathrm{MHz}$ flux densities to estimate the core spectral index, we obtain a flux density of $\sim 142 \mathrm{mJy}$ at the redshifted $21-\mathrm{cm}$ line frequency of $\sim 927 \mathrm{MHz}$. If the DLA covers only the core, the spin temperature of the absorber is $T_{\mathrm{s}} \sim 20 \mathrm{~K}$, a remarkably small value. However, the $21-\mathrm{cm}$ absorption is spread over $\sim 40 \mathrm{~km} \mathrm{~s}^{-1}$ and is hence unlikely to arise from a single line of sight. Further, the $408 \mathrm{MHz}$ Merlin map (Saikia et al. 1990) shows that the entire flux at this frequency is contained within $\sim 3 \operatorname{arcsecs}(\sim 16.8 \mathrm{kpc}$ at $z=0.5318)$. It is thus possible that the DLA covers some of the extended flux of the background source, besides the core. VLBI observations in the redshifted $21-\mathrm{cm}$ line would be necessary to detect the source components towards which the absorption occurs, and hence, the covering factor. For now, we note that a covering factor of unity results in $T_{\mathrm{s}}=310 \mathrm{~K}$; this is a definite upper limit on the spin temperature, which must then lie between $20 \mathrm{~K}$ and $310 \mathrm{~K}$.

9. PKS 2128-123 has a very flat spectrum between $8.4 \mathrm{GHz}$ and $750 \mathrm{MHz}$, with most measurements between these frequencies yielding a flux density of around $2 \mathrm{Jy}$ (see the $\mathrm{NED}^{1}$ for details). VLBA maps at 2.3 and $8.4 \mathrm{GHz}$ show that most of this flux is contained within $\sim 20$ milli-arcseconds, implying that the covering factor is likely to be close to unity. The HI column density of the $z \sim 0.4298$ absorber is $N_{\mathrm{HI}}=2.5 \pm$ $0.06 \times 10^{19} \mathrm{~cm}^{-2}$ (Ledoux et al. 2002); the $3 \sigma$ lower limit on the spin temperature is then $T_{\mathrm{s}}(3 \sigma)>980 \mathrm{~K}\left(\Delta V=7 \mathrm{~km} \mathrm{~s}^{-1}\right)$.

10. Finally, TXS $2342+342$ has a fairly flat spectrum between $1.4 \mathrm{GHz}$ and $8 \mathrm{GHz}$ (Wilkinson et al. 1998; White \& Becker 1992), indicating that the quasar is very compact at these frequencies. However, as Carilli et al. (1996) point out, the spectrum rises slowly down to the redshifted $21-\mathrm{cm}$ frequency of $356 \mathrm{MHz}$, indicating the presence of a more extended source component. Carilli et al. (1996) argue that the DLA is likely to at least cover the compact component and the covering factor is hence larger than 0.5 ; it is, however, unclear whether the extended flux is also covered. We will use $f=1$ in the analysis (following Carilli et al. 1996); the HI column density of $N_{\mathrm{HI}}=2 \pm 0.5 \times 10^{21} \mathrm{~cm}^{-2}$ then yields $T_{\mathrm{s}}(3 \sigma)>3585 \mathrm{~K}$ $\left(\Delta V=15 \mathrm{~km} \mathrm{~s}^{-1}\right)$. Carilli et al. (1996) estimated $T_{\mathrm{s}}>1800 \mathrm{~K}$ for this DLA.

\section{Discussion}

Neutral gas in the galaxy is believed to exist in two stable phases, a cold dense phase (the cold neutral medium, CNM), with typical temperatures of $\sim 100 \mathrm{~K}$, and a warm rarefied phase (the warm neutral medium, WNM), with typical temperatures of $\sim 8000 \mathrm{~K}$. It should be emphasised that such a multi-phase medium exists only over a finite range of interstellar pressures (Wolfire et al. 1995); at higher pressures only the CNM phase exists, while at lower pressures only the WNM phase is found. Further, although the multi-phase structure results from a balance between the heating and cooling rates, which themselves depend on various quantities such as the radiation field, the metallicity, the dust content, etc., it has been

\footnotetext{
1 NASA/IPAC Extragalactic Database.
} 
Table 3. Damped Ly- $\alpha$ systems with $21-\mathrm{cm}$ observations.

\begin{tabular}{|c|c|c|c|c|c|c|c|c|c|}
\hline Name & $z_{\mathrm{abs}}$ & $\begin{array}{c}N_{\mathrm{HI}} \\
10^{20} \mathrm{~cm}^{-2}\end{array}$ & $f$ & $\begin{array}{c}(1 / f) \int \tau \mathrm{d} V \\
\mathrm{~km} \mathrm{~s}^{-1}\end{array}$ & $\begin{array}{c}\Delta V_{21}{ }^{a} \\
\mathrm{~km} \mathrm{~s}^{-1}\end{array}$ & $\begin{array}{c}T_{\mathrm{s}}^{b} \\
\mathrm{~K}\end{array}$ & $\begin{array}{l}\text { Absorber } \\
\text { ID }\end{array}$ & $\begin{array}{c}b^{d} \\
\mathrm{kpc}\end{array}$ & Refs. \\
\hline $0738+313(\mathrm{~A})$ & 0.09123 & $15 \pm 2$ & 1 & $1.0 \pm 0.01$ & 40 & $825 \pm 120$ & Dwarf & $<4$ & $1,2,3$ \\
\hline PKS 0439-433c & 0.10097 & $?$ & 1 & $0.07 \pm 0.02$ & 10 & $?$ & Spiral? & 8.0 & 4,5 \\
\hline $0738+313(\mathrm{~B})$ & 0.22125 & $7.9 \pm 1.4$ & 1 & $0.488 \pm 0.004$ & 30 & $890 \pm 165$ & Dwarf & 20 & $1,3,6$ \\
\hline PKS 0952+179 & 0.23779 & $21 \pm 2.5$ & 0.25 & $0.56 \pm 0.02$ & 30 & $2055 \pm 330$ & LSB & $<7$ & $7,8,9$ \\
\hline PKS $1413+135^{c \star}$ & 0.24671 & $200^{\star}$ & 0.12 & 59.43 & 39 & 185 & Spiral & $?$ & $10,11,12$ \\
\hline PKS 1127-145 & 0.3127 & $51 \pm 9$ & 1 & $3.074 \pm 0.002$ & 120 & $910 \pm 160$ & LSB & $<10$ & $8,9,13$ \\
\hline PKS 1229-021 & 0.39498 & $5.6 \pm 0.5$ & 0.5 & $1.81 \pm 0.01$ & 110 & $170 \pm 15$ & Spiral & 11 & $14,15,16$ \\
\hline QSO $0248+430$ & 0.3941 & $?$ & $?$ & 2.99 & 40 & $?$ & - & - & 17 \\
\hline PKS 2128-123 & 0.4298 & $0.25 \pm 0.06$ & 1 & $<0.014$ & - & $>980$ & - & - & 18,19 \\
\hline 3C196 & 0.43670 & $6.3 \pm 1.6$ & $?$ & $?$ & 250 & - & Spiral & 9.2 & $14,20,21$ \\
\hline PKS 1243-072 & 0.4367 & $?$ & 1 & 0.38 & 23 & $?$ & Spiral & 11.3 & 17,22 \\
\hline $3 \mathrm{C} 446^{c}$ & 0.4842 & 6.3 & 0.1 & $<0.22$ & - & $>1570$ & - & - & 13,23 \\
\hline A0 $0235+164$ & 0.524 & 50 & 1 & $13 \pm 0.6$ & 125 & 210 & Spiral & $<11.2$ & $24,25,26$ \\
\hline B2 $0827+243$ & 0.5247 & $2.0 \pm 0.2$ & 0.67 & $0.33 \pm 0.03$ & 50 & $330 \pm 70$ & Spiral & 34 & $7,8,9$ \\
\hline PKS $1629+12$ & 0.5318 & 2.8 & 1 & $0.494 \pm 0.001$ & 40 & 310 & Spiral & 17 & 9,19 \\
\hline PKS $0118-272^{c}$ & 0.5579 & $?$ & 0.5 & $<0.12$ & - & $?$ & - & - & 7,27 \\
\hline $3 \mathrm{C} 286$ & 0.69215 & $20 \pm 1.6$ & $1 ?$ & $0.91 \pm 0.09$ & 18 & $1205 \pm 240$ & LSB & 19 & $15,28,29$ \\
\hline PKS 0454+039 & 0.8596 & $5 \pm 0.16$ & 1 & $<0.32$ & - & $>855$ & Dwarf & 6.4 & $15,30,31$ \\
\hline PKS $0215+015^{c}$ & 1.3449 & 0.8 & 1 & $<0.043$ & - & $>1020$ & - & - & 19,32 \\
\hline QSO $0957+561 \mathrm{~A}$ & 1.3911 & $2.1 \pm 0.5$ & $?$ & - & - & - & - & - & 8,19 \\
\hline PKS 1354+258 & 1.4205 & $32 \pm 2$ & - & - & - & - & - & - & 8,19 \\
\hline MC3 1331+170 & 1.77636 & $15 \pm 1.5$ & 0.8 & $0.5 \pm 0.1$ & 25 & $1645 \pm 615$ & - & - & $31,33,34$ \\
\hline PKS 1157+014 & 1.944 & $63 \pm 10$ & 0.55 & $4 \pm 0.2$ & 60 & $865 \pm 190$ & - & - & $31,35,36$ \\
\hline PKS 0458-02 & 2.03945 & $45 \pm 8$ & 1 & $6.4 \pm 0.4$ & 30 & $385 \pm 100$ & - & - & $34,37,47$ \\
\hline PKS 0528-2505 & 2.8110 & $22 \pm 1$ & 1 & $<1.70$ & - & $>710$ & - & - & $38,39,40$ \\
\hline TXS 2342+342 & 2.9084 & $20 \pm 0.5$ & 1 & $<0.306$ & - & $>3585$ & - & - & 19,41 \\
\hline PKS 1354-107 & 2.966 & 6 & 1 & $<0.345$ & - & $>955$ & - & - & 19,42 \\
\hline PKS 0537-286 & 2.9742 & 2 & 1 & $<0.058$ & - & $>1890$ & - & - & 19,42 \\
\hline PKS 0336-014 & 3.0619 & $16 \pm 1.3$ & 1 & $<0.095$ & - & $>9240$ & - & - & $19,41,46$ \\
\hline PKS 0335-122 & 3.178 & $6 \pm 1$ & 1 & $<0.084$ & - & $>3920$ & - & - & 19,42 \\
\hline PKS 0201+113 & 3.3875 & $18 \pm 3$ & 1 & $<0.30$ & - & $>3290$ & - & - & $41,44,45$ \\
\hline
\end{tabular}

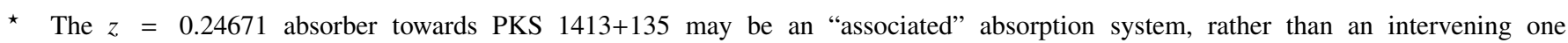
(Perlman et al. 2002). Further, its HI column density is estimated from an X-ray spectrum (Stocke et al. 1992).

${ }^{a}$ The velocity width $\Delta V$ quoted here is the entire velocity range over which absorption is seen.

${ }^{b}$ The spin temperature values (or $3 \sigma$ limits) quoted here have been consistently (re-)computed from the published data using Eq. (1), and the latest available values for all parameters in the equation. The values quoted here and the errors have been rounded off to the nearest $5 \mathrm{~K}$. The errors on $N_{\mathrm{HI}}$ and hence, on $T_{\mathrm{s}}$, have been quoted when available in the original references; in the case of asymmetric errors, the larger value has been quoted. In certain instances, the values may differ slightly from that quoted by the authors in the original reference. The only exception is PKS 0201+113, for which multiple and quite different values are quoted in the literature. We use the value from Kanekar \& Chengalur (1997), modified by the new HI column density of $1.8 \pm 0.3 \times 10^{21} \mathrm{~cm}^{-2}$, from Ellison et al. (2001b).

${ }^{c}$ Candidate damped Lyman- $\alpha$ systems, based on IUE spectra, metal lines or X-ray spectra.

${ }^{d}$ The impact parameter $b$ has been calculated using an $\Omega=1, \Lambda=0$ Universe, with $H_{0}=65 \mathrm{~km} \mathrm{~s}^{-1} \mathrm{Mpc}^{-1}$.

${ }^{1}$ Rao \& Turnshek (1998), ${ }^{2}$ Chengalur \& Kanekar (1999), ${ }^{3}$ Cohen (2001), ${ }^{4}$ Petitjean et al. (1996), ${ }^{5}$ Kanekar et al. (2001a), ${ }^{6}$ Kanekar et al. (2001b), ${ }^{7}$ Kanekar \& Chengalur (2001), ${ }^{8}$ Rao \& Turnshek (2000), ${ }^{9}$ Nestor et al. (2001), ${ }^{10}$ Stocke et al. (1992), ${ }^{11}$ Carilli et al. (1992), ${ }^{12}$ Perlman et al. (2002). ${ }^{13}$ Chengalur \& Kanekar (2000), ${ }^{14}$ Kanekar \& Chengalur (2002), ${ }^{15}$ Boisse et al. (1998), ${ }^{16}$ Le Brun et al. (1997), ${ }^{17}$ Lane \& Briggs (2002), ${ }^{18}$ Ledoux et al. (2002), ${ }^{19}$ This paper, ${ }^{20}$ Briggs et al. (2001), ${ }^{21}$ Cohen et al. (1996), ${ }^{22}$ Kanekar et al. (2002), ${ }^{23}$ Lanzetta et al. (1995), ${ }^{24}$ Roberts et al. (1976), ${ }^{25}$ Burbidge et al. (1996), ${ }^{26}$ Cohen et al. (1999), ${ }^{27}$ Vladilo et al. (1997), ${ }^{28}$ Steidel et al. (1994), ${ }^{29}$ Davis \& May (1978), ${ }^{30}$ Steidel et al. (1995), ${ }^{31}$ Briggs et al. (1983), ${ }^{32}$ Turnshek \& Rao (2002), ${ }^{33}$ Wolfe \& Davis (1979), ${ }^{34}$ Pettini et al. (1994), ${ }^{35}$ Briggs et al. (1984), ${ }^{36}$ Wolfe et al. (1981), ${ }^{37}$ Wolfe et al. (1985), ${ }^{38}$ Carilli et al. (1996), ${ }^{39}$ Morton et al. (1980), ${ }^{40}$ Foltz et al. (1988), ${ }^{41}$ White et al. (1993), ${ }^{42}$ Ellison et al. (2001a), ${ }^{43}$ Ellison et al. (2001b), ${ }^{44}$ Kanekar \& Chengalur (1997), ${ }^{45}$ Briggs et al. (1997), ${ }^{46}$ Prochaska et al. (2001), ${ }^{47}$ Prochaska \& Wolfe (1999). 
shown that a two-phase pressure equilibrium is possible over fairly wide ranges of the above quantities (and, of course, the interstellar pressure; Wolfire et al. 1995). On the observational front, the existence of neutral gas in a multi-phase medium has been established not just for gas in the solar neighborhood, but also in high velocity clouds (e.g. Braun \& Burton 2000), dwarf galaxies (e.g. Young \& Lo 1997) and DLAs (Lane et al. 2000; Kanekar et al. 2001b). In the latter case of the two DLAs at $z=0.0912$ and $z=0.2212$ toward $\mathrm{B} 0738+313$, it has been explicitly shown that the observed high spin temperatures are a consequence of $70-80 \%$ of the gas being in the warm phase. In this regard, the multi-phase structure of at least these two systems is more like that of dwarf galaxies (see, for example, Young \& Lo 1997) than large spiral disks.

Table 3 contains a list of the 31 known candidate and confirmed DLAs for which $21-\mathrm{cm}$ observations are available in the published literature (this is an update of the list in Chengalur \& Kanekar 2000). Note that the Lyman- $\alpha$ line has so far not been observed for the DLAs at $z=$ 0.24671 towards PKS $1413+135, z=0.3941$ towards QSO $0248+430$ and $z=0.43669$ towards PKS 1243-072; however, all three systems show strong $21-\mathrm{cm}$ absorption, implying (for reasonable values of $T_{\mathrm{s}}$ ) HI column densities higher than $10^{20} \mathrm{~cm}^{-2}$ (Carilli et al. 1992; Kanekar et al. 2002; Lane \& Briggs 2002) and have hence been included in the present sample. Further, the $z=0.24671$ absorber towards PKS $1413+135$ may be an associated system (Perlman et al. 2002); the HI column density listed in Table 3 is estimated from the deficit of soft X-rays (Stocke et al. 1992). No similar direct estimate exists for the HI column densities of the systems towards PKS 1243-072 and QSO $0248+430$. We also note that two candidate DLAs (on the basis of metal lines), at $z=0.5579$ towards PKS 0118-272 (Kanekar \& Chengalur 2001) and $z=0.101$ towards PKS 0439-433 (Kanekar et al. 2001a) have, for completeness, been included in this table; however, they will not be used in the later analysis, as their HI column densities (and hence, spin temperatures) are unknown. Columns 3-7 of the table contain the HI column density (in units of $10^{20} \mathrm{~cm}^{-2}$ ), the adopted covering factor $f$, the "true" equivalent width $\left((1 / f) \int \tau \mathrm{d} V\right)$, the velocity spread of $21-\mathrm{cm}$ absorption (between nulls, in $\mathrm{km} \mathrm{s}^{-1}$ ) and the spin temperature. Besides these, Cols. 8 and 9 list the nature of the objects identified as the DLA hosts and their impact parameters $(b$, in $\mathrm{kpc}$ ) to the QSO line of sight. Of course, it is possible that the latter identifications are in error in some cases if, for example, the absorption arises in an object closer to the QSO but too faint to be identified in optical/IR images. With this caveat in mind, we note that seven of the DLA hosts have been identified with large, luminous galaxies, with luminosities close to $L_{\star}$.

Figure 3 shows a plot of the measured spin temperatures as a function of redshift for the 24 DLAs of the complete sample which have reliable estimates of the spin temperature (note that this plot includes the $z=0.24671$ absorber towards PKS 1413+135 which may be an associated system). The seven systems in Table 3 which are not included in Fig. 3 are the two 21-cm absorbers towards PKS 1243-072 and QSO 0248+430

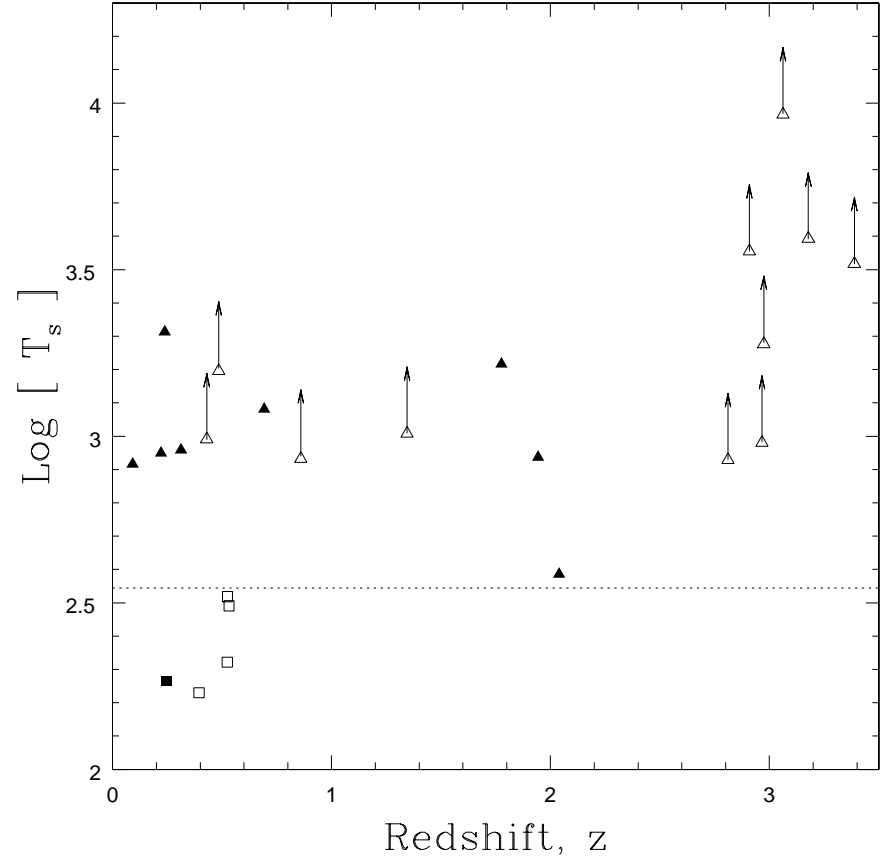

Fig. 3. The spin temperature $\left(\log \left[T_{\mathrm{s}}\right]\right)$ of the 24 DLAs of the complete sample with $T_{\mathrm{s}}$ estimates as a function of redshift $z$. Note that seven DLAs listed in Table 3 have not been included here for reasons listed in the text. Objects identified with spiral galaxies are plotted using squares; the filled square shows the $z=0.24671$ spiral towards PKS $1413+135$, which may be an associated system. Other detections are shown as filled triangles, with non-detections shown as open triangles, with arrows.

for which the HI column density is unknown (see above), the DLAs towards PKS $1354+258$ and QSO $0957+561$ for which the covering factor is highly uncertain (see Sect. 4), the two candidate DLAs towards PKS 0118-272 and PKS 0439-433 and, finally, the $z=0.437$ absorber towards 3C196, where the lines of sight to the optical QSO and the radio continuum trace very different paths through the absorbing galaxy. The dotted line in Fig. 3 is at $350 \mathrm{~K}$ - more than $80 \%$ of spin temperature measurements in M 31 and the Milky Way have values $<350 \mathrm{~K}$ (Braun \& Walterbos 1992). It can be seen that the spin temperatures of the majority of DLAs are considerably higher than those typical of lines of sight through the disks of the Milky Way or nearby spiral galaxies. The only exceptions to the above are the five systems at low $z$ which have been identified with spiral disks and the DLA at $z \sim 2.04$ towards PKS 0458-020, for which $T_{\mathrm{s}} \sim 385 \pm 100 \mathrm{~K}$; we discuss the latter separately below. Note that it is not presently possible to estimate the spin temperatures of the remaining two $L \sim L_{\star}$ DLAs at $z \sim 0.437$ towards PKS 1243-072 and 3C196. Finally, the 21-cm absorption profile for PKS 1629+12 reported in this paper continues the trend (noted earlier in Chengalur \& Kanekar 2000; Kanekar \& Chengalur 2001) of low spin temperatures being found in all DLAs identified as $L \sim L_{\star}$ galaxies.

Figure 4 presents the data in an alternative way, as a scatter plot of the "true" HI 21-cm equivalent width (i.e. $(1 / f) \int \tau \mathrm{d} V$ ) against the HI column density. Both of these are directly observable quantities. The crosses show the same quantities 


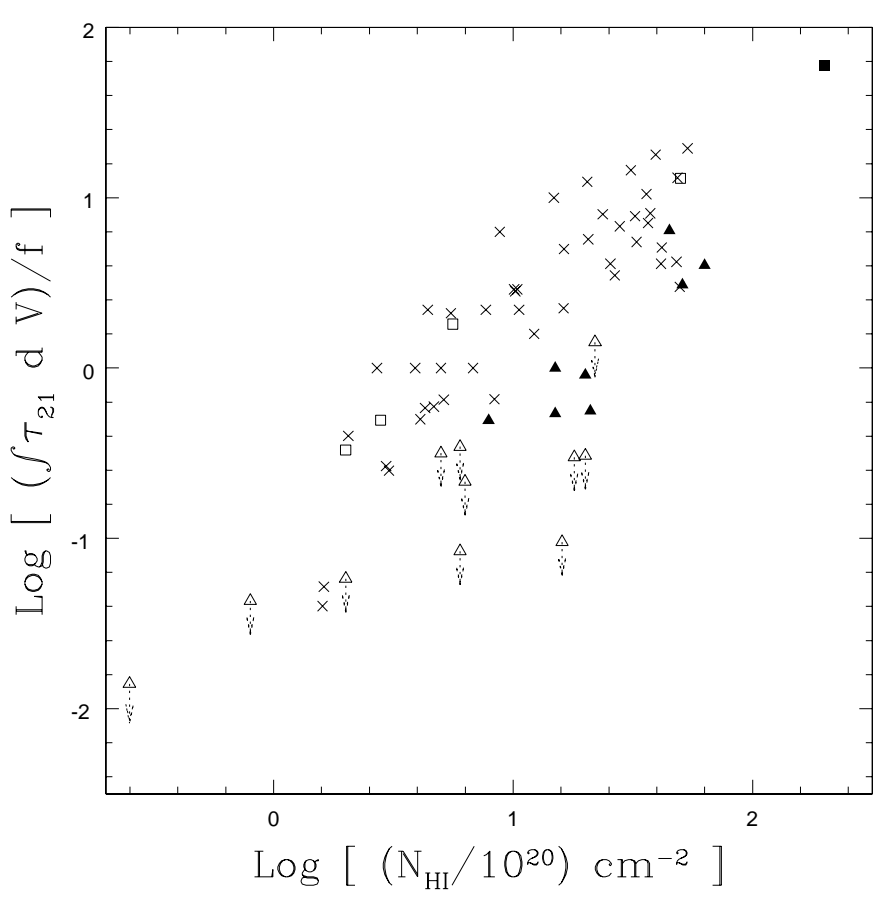

Fig. 4. The true equivalent width $\left(\int \tau \mathrm{d} V / f\right)$ as a function of the HI column density for the 24 DLAs of the complete sample for which we can calculate $T_{\mathrm{s}}$ values. Objects identified with spiral galaxies are plotted using squares; the filled square shows the $z=0.24671$ spiral towards PKS $1413+135$, which may be an associated system. Other detections are shown as filled triangles, with non-detections shown as open triangles, with arrows. The crosses show measurements for galactic HI by Colgan et al. (1988) and Payne et al. (1982), using 21-cm emission/absorption studies.

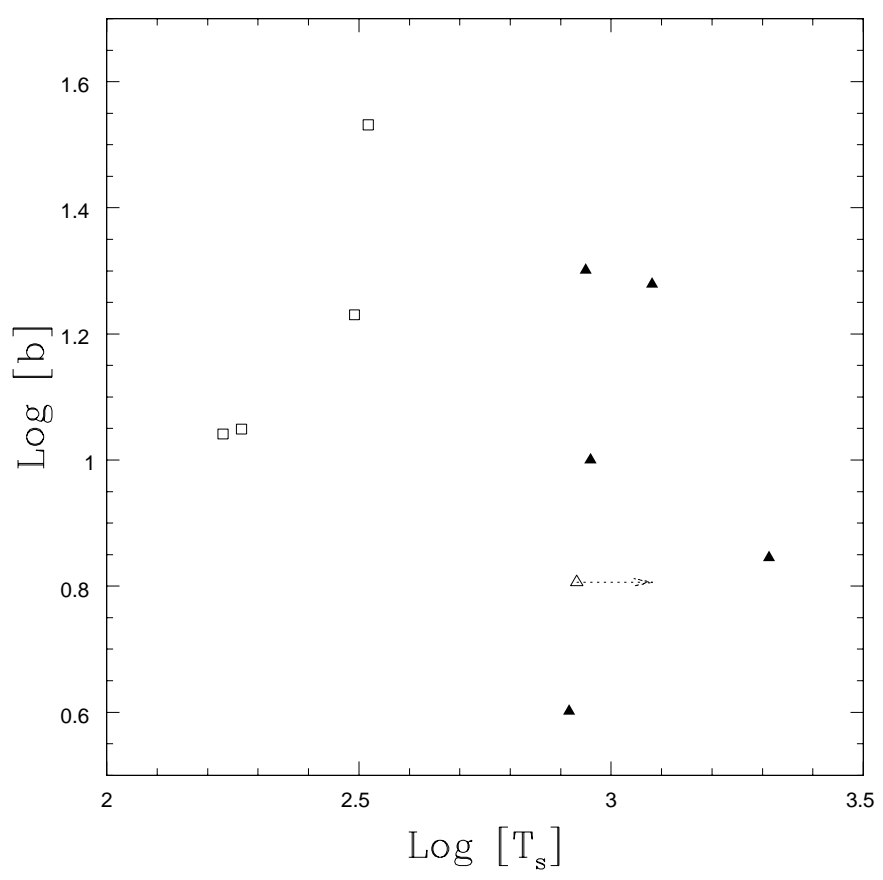

Fig. 5. The impact parameter $b$ (in kpc) as a function of spin temperature for 10 DLAs at $z<1$. The open squares represent objects identified with spiral galaxies; the remaining detections are shown as filled triangles, with non-detections shown as open triangles, with arrows. for Galactic HI clouds (Colgan et al. 1988; Payne et al. 1982), measured using 21-cm absorption/emission studies. HI column density estimates using observations of the Lyman- $\alpha$ line have been found to be in good agreement with estimates from $21-\mathrm{cm}$ emission studies (Dickey \& Lockman 1990). Figure 4 hence allows a direct comparison of the same observables for DLAs and galactic clouds. It can be clearly seen that, at a given HI column density, the majority of DLAs tend to have smaller $21-\mathrm{cm}$ equivalent widths than those found for lines of sight through the Galaxy. The only exceptions are DLAs that are known to be associated with spiral galaxies (shown as squares) and the $z \sim 2.04$ DLA towards PKS 0458-020, (which we discuss in more detail below), which do lie within the range of Galactic values. The conclusion would seem to be that the majority of DLAs contain larger fractions of the warm (and thus weakly absorbing) phase of neutral hydrogen than typical in nearby spirals, as earlier pointed out by Carilli et al. (1996) and Kanekar \& Chengalur (2001).

It is possible that the high $T_{\mathrm{s}}$ values seen in DLAs might arise due to absorption originating in the outskirts of large disks, which would also provide a higher absorption crosssection than the inner regions of these galaxies. Figure 5 shows a plot of $T_{\mathrm{s}}$ versus impact parameter $b$ for the 10 DLAs of the sample (all with $z<1$ ) with optical identifications, as well as estimates of both $T_{\mathrm{s}}$ and impact parameter; absorbers identified with spiral galaxies are shown as open squares. No correlation can be seen to exist between $T_{\mathrm{s}}$ and $b$. On the contrary, the largest impact parameter of the sample $(b=34 \mathrm{kpc})$ is seen in a low $T_{\mathrm{s}}$ system, the $z=0.5247$ DLA towards B2 $0827+243$, while four of the high $T_{\mathrm{s}}$ DLAs have $b<10 \mathrm{kpc}$. The low redshift sample clearly does not support the hypothesis that the high $T_{\mathrm{s}}$ seen in low redshift DLAs is because the absorption arises from lines of sight which pass through the outer regions of disk galaxies. Instead, it appears that the spin temperature correlates with the nature of the absorbing galaxy. We discuss next the $z \sim 2.04$ DLA towards PKS 0458-020, the only high $z$ DLA with a spin temperature $T_{\mathrm{s}}<400 \mathrm{~K}$.

The $z=2.03945$ DLA towards PKS 0458-020 is as yet the highest redshift DLA with a confirmed detection of 21-cm absorption (Wolfe et al. 1985). Briggs et al. (1989) found the VLBI 21-cm absorption profile to be very similar to the single dish profile and used this to argue that the absorber shows little structure on the scale of the extended radio continuum emission of the background source; these observations hence imply that the transverse size of the absorber is larger than $8 h^{-1} \mathrm{kpc}$. Further, the low ionization metal lines of the DLA are clearly asymmetric (Prochaska \& Wolfe 1997), which can be explained if the absorber is a rapidly rotating disk (although such asymmetries are also explanable in other models; see, for example, Haehnelt et al. 1998, McDonald \& Miralda-Escude 1999). The spin temperature of the absorber was originally estimated to be $T_{\mathrm{s}}=594 \pm 297 \mathrm{~K}$ (Wolfe et al. 1985), the large error arising due to uncertainties in fitting the Lyman- $\alpha$ profile $\left(N_{\mathrm{HI}}=8 \pm 4 \times\right.$ $10^{21} \mathrm{~cm}^{-2}$; Wolfe et al. 1985). Recently, however, the HI column density has been measured far more accurately by Pettini et al. (1994), who quote $N_{\mathrm{HI}}=4.5 \pm 0.8 \times 10^{21} \mathrm{~cm}^{-2}$. Combining this with the HI $21-\mathrm{cm}$ absorption profile (and taking $f=1$, 
Table 4. The CNM fraction in high $z$ DLAs.

\begin{tabular}{lcccc}
\hline \hline Name & $z_{\text {abs }}$ & $\begin{array}{c}N_{\mathrm{HI}} \\
(200 \mathrm{~K}) \\
\times 10^{20}\end{array}$ & $\begin{array}{c}N_{\mathrm{HI}} \\
(\text { Total })\end{array}$ & $f_{\mathrm{CNM}}$ \\
& & $\times 10^{20}$ & \\
\hline PKS 0528-250 & 2.8110 & $<6.2$ & $22 \pm 1$ & $<0.28$ \\
TXS 2342+342 & 2.9084 & $<0.7$ & $20 \pm 0.5$ & $<0.03$ \\
PKS 1354-107 & 2.966 & $<1.3$ & 6.0 & $<0.21$ \\
PKS 0537-286 & 2.9742 & $<0.2$ & 2.0 & $<0.09$ \\
PKS 0336-014 & 3.0619 & $<0.2$ & $16 \pm 1.3$ & $<0.01$ \\
PKS 0335-122 & 3.178 & $<0.2$ & $6 \pm 1$ & $<0.04$ \\
PKS 0201+113 & 3.3875 & $<1.1$ & $18 \pm 3$ & $<0.06$ \\
\hline
\end{tabular}

as confirmed by the VLBI observations) yields $T_{\mathrm{s}}=385 \pm$ $100 \mathrm{~K}$, a value not too far from those obtained in local spirals; this is the first case of a DLA at $z \gtrsim 0.6$ with a spin temperature less than $400 \mathrm{~K}$. Interestingly enough, the $21-\mathrm{cm}$ equivalent width of the absorber too lies close to the range of Galactic values shown in Fig. 4. We note, however, that the metallicity of the absorber is far lower than solar values $([\mathrm{Zn} / \mathrm{H}]=$ $-1.16 \pm 0.09$; Prochaska \& Wolfe 1999), implying that the DLA has not undergone much chemical evolution. Detailed analysis of the formation of a multi-phase medium in protogalaxies (Spaans \& Carollo 1997; Spaans \& Norman 1997) indicate that the epoch of formation of a multi-phase ISM is a strong function of the galactic mass; galaxies with baryonic mass $\sim 5 \times 10^{11} M_{\odot}$ form the cold phase by $z \sim 3$ while dwarf galaxies, with baryonic mass $\sim 3 \times 10^{9} M_{\odot}$, typically form the cold phase only by $z \sim 1$. The relatively low measured spin temperature in the $z \sim 2.04$ DLA thus makes it likely that the absorber is a large galaxy. The VLBI observations and the low spin temperature are hence both consistent with the DLA being a massive galaxy, with the high mass providing a sufficiently high central pressure to compensate for the low metallicity and produce a sizeable fraction of the cold phase of HI.

The evolution of spin temperature with redshift has been the subject of interest for some time. The original observations of high spin temperatures at high redshift led to the suggestion that the elevation of the spin temperature was perhaps due to evolutionary effects. More recently, the detection of low redshift DLAs $(z \lesssim 0.2)$ with high $T_{\mathrm{s}}$ values (Lane et al. 1998; Chengalur \& Kanekar 1999) indicated that a simple trend did not exist in the evolution of $T_{\mathrm{s}}$ with redshift. However, Chengalur \& Kanekar (2000) noted the following trend: DLAs with both low and high spin temperatures are found at low redshifts $(z \lesssim 1)$, while only high $T_{\mathrm{s}}$ DLAs are found at high redshifts $(z \gtrsim 3)$. The present, larger sample of Fig. 3 shows this trend clearly, with the exceedingly high values of spin temperature at $z \gtrsim 3$ indicating that these systems contain very small fractions of the CNM phase of HI.

The non-detection of $\mathrm{HI}$ absorption in the present fairly deep search can be used to place stringent constraints on the fractional content of CNM in the high $z$ absorbers. To do so, we note that the allowed values of CNM temperatures in the Milky Way lie between $40 \mathrm{~K}$ and $200 \mathrm{~K}$ (Wolfire et al. 1995). We hence use $200 \mathrm{~K}$ (in Eq. (1)) as an upper limit to the temperature of the CNM phase in the seven $z \gtrsim 3$ DLAs to estimate upper limits on the CNM fraction in these systems. The original high resolution spectra (see Table 1) are used for this purpose, not the smoothed spectra used to estimate the spin temperature. The results are shown in Table 4 where the third column gives the $3 \sigma$ upper limit to the HI column density in $200 \mathrm{~K}$ gas (note that this limit scales linearly with the assumed CNM temperature and would hence be even lower for lower temperature $\mathrm{CNM}$ ). The last column in this table gives the upper limit to the fraction of the $\mathrm{HI}$ in $\mathrm{CNM}, f_{\mathrm{CNM}}$; it can be seen that five of the seven systems at $z \gtrsim 3$ have CNM fractions less than $10 \%$, with three of these having $f_{\mathrm{CNM}}<5 \%$. Clearly, most of the gas in the high $z$ DLAs is warm, the typical fraction of CNM gas is even smaller than that in the two high $T_{\mathrm{s}}$ DLAs at low redshift with measured CNM fractions (Lane et al. 2000; Kanekar et al. 2001b) or in local dwarf galaxies (Young \& Lo 1997).

An independent line of evidence for high temperatures at high redshift comes from observations of the $n(\mathrm{CII}) / n(\mathrm{CI})$ ratio (Liszt 2002), which is quite different in the CNM and the WNM. Liszt (2002) used observations of this ratio in 11 DLAs to suggest that high redshift $(z>2.3)$ DLAs are dominated by warm gas, with at most a few per cent of the neutral HI in the CNM. This is in agreement with our results. Besides this, it has been observed that high $z$ DLAs typically have very low molecular gas fractions; this can be understood in models in which the absorbing gas is at high temperature (Petitjean et al. 2000; but see also Liszt 2002). We note, in passing, that it is exceedingly interesting that the $z=3.0619$ DLA towards PKS 0336-014 has $T_{\mathrm{s}}>9240 \mathrm{~K}$, implying that its gas is significantly hotter than even the standard WNM in the Milky Way (which has temperatures in the range $5000-8000 \mathrm{~K}$; Wolfire et al. 1995).

Of the 24 DLAs with $T_{\mathrm{s}}$ measurements, 16 are at $z<2$ and 8 at $z>2$; we will call these the low and high $z$ samples respectively. The sample is of a sufficiently large size to carry out a statistical comparison to test the likelihood that the systems in the two samples stem from the same parent distribution. The Gehan test (Gehan 1965; Miller 1981) is appropriate for this comparison as it can be directly used in the case of limits on the measured quantity (in our case, the spin temperature). When applied to our data, this test rules out the possibility of the two samples being drawn from the same distribution at the $\sim 99 \%$ confidence level. (If one chooses to treat the $3 \sigma$ upper limits as detections, a Kolmogorov-Smirnov rank 1 test rules out the above hypothesis at the $95 \%$ confidence level.) It is thus very unlikely that the high and low $z$ DLAs are systems of similar "character". We note that $z=2$ was used as the redshift of separation of the two samples as this is the approximate redshift where star formation is believed to peak; it is hence plausible that systems at $z>2$ will show differences from those at lower redshifts. Since we have only 4 systems with $T_{\mathrm{s}}$ estimates in the redshift range $1<z<2.8$ (the $z \sim 1.4$ DLAs towards PKS 1354+258 and QSO 0957+561A do not have good estimates of $T_{\mathrm{s}}$ ), it is not possible to independently determine the threshold redshift with the present sample.

In conclusion, the sample of damped Lyman- $\alpha$ systems which have been searched for 21-cm absorption now consists of 31 systems; estimates of the spin temperature are available 
in 24 cases, of which 16 DLAs are at $z<2$ and 8 at $z>2$. All of the low $T_{\mathrm{s}}$, low $z$ DLAs have been identified with large, luminous galaxies, while all DLAs at $z<1$ with high spin temperature $\left(T_{\mathrm{s}} \gtrsim 1000 \mathrm{~K}\right)$ have been identified either with LSBs or dwarfs. It thus appears likely that the spin temperature depends mainly on the nature of the absorbing galaxy, as suggested earlier by Chengalur \& Kanekar (2000). We use a recent measurement of the HI column density in the $z=2.03945$ DLA towards PKS 0458-020 to derive a relatively low $T_{\mathrm{s}}$ value in the absorber; this is the first high $z$ DLA to show $T_{\mathrm{s}}<400 \mathrm{~K}$. The low metallicity allied with a low spin temperature suggest that the DLA is a massive galaxy, consistent with VLBI limits of its transverse size. The new deep GMRT 21-cm spectra of this paper have also been used to estimate the fraction of $\mathrm{HI}$ in the cold phase, $f_{\mathrm{CNM}}$, in the absorbers at $z \gtrsim 3$; it was found that $f_{\mathrm{CNM}}<0.3$ in all seven absorbers, with $f_{\mathrm{CNM}}<0.1$ in five of the seven cases. These results are in good agreement with estimates of $f_{\mathrm{CNM}} \sim f e w \%$, from observations of the $n(\mathrm{CII}) / n(\mathrm{CI})$ ratio (Liszt 2002). Finally, we have used the above spin temperature estimates to test the likelihood that the $z>2$ and $z<2$ DLAs are drawn from the same parent population and rule out this hypothesis at the $\sim 99 \%$ confidence level.

Acknowledgements. We thank the referee, Wendy Lane, for numerous suggestions and comments on an earlier version which have significantly improved this paper. These observations would not have been possible without the many years of dedicated effort put in by the GMRT staff in order to build the telescope. The GMRT is operated by the National Centre for Radio Astrophysics of the Tata Institute of Fundamental Research. This research has made use of the United States Naval Observatory (USNO) Radio Reference Frame Image Database (RRFID) and the NASA/IPAC Extragalactic Database (NED) which is operated by the Jet Propulsion Laboratory, California Institute of Technology, under Contract with the National Aeronautics and Space Administration.

\section{References}

Aldcroft, T. L., Bechtold, J., \& Elvis, M. 1994, ApJS, 93, 1 Barthel, P. D., Tytler, D. R., \& Thomson, B. 1990, A\&AS, 82, 339

Boissé, P., le Brun, V., Bergeron, J., \& Deharveng, J.-M. 1998, A\&A, 333, 841

Braun, R., \& Walterbos, R. A. M. 1992, ApJ, 386, 120

Braun, R., \& Burton, W. B. 2000, A\&A, 354, 853

Briggs, F. H., \& Wolfe, A. M. 1983, ApJ, 268, 76

Briggs, F. H., Turnshek, D. A., \& Wolfe, A. M. 1984, ApJ, 287, 549

Briggs, F. H., Wolfe, A. M., Liszt, H. S., Davis, M. M., \& Turner, K. L. 1989, ApJ, 341, 650

Briggs, F. H., Brinks, E., \& Wolfe, A. M. 1997, AJ, 113, 467

Briggs, F. H., de Bruyn, A. G., \& Vermeulen, R. C. 2001, A\&A, 373, 113

le Brun, V., Bergeron, J., Boissé, P., \& Deharveng, J.-M. 1997, A\&A, 321, 733

Burbidge, E. M., Beaver, E. A., Cohen, R. D., Junkkarinen, V. T., \& Lyons, R. W. 1996, AJ, 112, 2533

Campbell, R. M., Lehar, J., Corey, B. E., Shapiro, I. I., \& Falco, E. E. 1995, AJ, 110, 2566

Carilli, C. L., Perlman, E. S., \& Stocke, J. T. 1992, ApJ, 400, L13

Carilli, C. L., Lane, W., de Bruyn, A. G., Braun, R., \& Miley, G. K. 1996, AJ, 111, 1830

Cohen, R. D., Beaver, E. A., Diplas, A., et al. 1996, ApJ, 456, 132
Cohen, R. D., Burbidge, E. M., Junkkarinen, V. T., Lyons, R. W., \& Madejski, G. 1999, BAAS, 194, 7101

Cohen, J. G. 2001, AJ, 121, 2895

Colgan, S. W. J., Salpeter, E. E., \& Terzian, Y. 1988, ApJ, 328, 275

Condon, J. J., Cotton, W. D., Greisen, E. W., et al. 1998, AJ, 115, 1693

Chengalur, J. N., \& Kanekar, N. 1999, MNRAS, 302, L29

Chengalur, J. N., \& Kanekar, N. 2000, MNRAS, 318, 303

Dallacasa, D., Bondi, M., Alef, W., \& Mantovani, F. 1998, A\&AS, 129,219

Davis, M. M., \& May, L. S. 1978, ApJ, 219, 1

Dickey, J., \& Lockman, F. J. 1990, ARA\&A, 28, 215

Ellison, S. L., Yan, L., Hook, I. M., et al. 2001a, A\&A, 379, 393

Ellison, S. L., Pettini, M., Steidel, C. C., \& Shapley, A. E. 2001b, ApJ, 549,770

Foltz, C. B., Chaffee, Jr. F. H., \& Black, J. H. 1988, ApJ, 324, 267

Fomalont, E. B. 2000, ApJS, 131, 95

Garrett, M. A., Calder, R. J., Porcas, R. W., et al. 1994, MNRAS, 270, 457

Gehan, E. A. 1965, Biometrika, 52, 203

Griffith, M. R., Wright, A. E., Burke, B. F., \& Ekers, R. D. 1994, ApJS, 90, 179

Gurvits, L. I., Schilizzi, R. T., Barthel, P. D., et al. 1994, A\&A, 291, 737

Haehnelt, M. G., Steinmetz, M., \& Rauch, M. 1998, ApJ, 495, 64

Hirabayashi, H., Fomalont, E. B., Horiuchi, S., et al. 2000, PASJ, 52, 997

Kanekar, N., \& Chengalur, J. N. 1997, MNRAS, 292, 831

Kanekar, N., Chengalur, J. N., Subrahmanyan, R., \& Petitjean, P. 2001a, A\&A, 367, 46

Kanekar, N., Ghosh, T., \& Chengalur, J. N. 2001b, A\&A, 373, 394

Kanekar, N., \& Chengalur, J. N. 2001, A\&A, 369, 42

Kanekar, N., Athreya, R., \& Chengalur, J. N. 2002, A\&A, 382, 838

Kanekar, N., \& Chengalur, J. N. 2002, in preparation

Lane, W., Smette, A., Briggs, F. H., et al. 1998, AJ, 116, 26

Lane, W., Briggs, F. H., \& Smette, A. 2000, ApJ, 532, 146

Lane, W., \& Briggs, F. H. 2002, ApJ, 561, L27

Lanzetta, K. M., Wolfe, A. M., \& Turnshek, D. A. 1995, ApJ, 440, 435

Ledoux, C., Bergeron, J., \& Petitjean, P. 2002, A\&A, 385, 802

Liszt, H. 2002, A\&A, 389, 393

McDonald, P., \& Miralda-Escude, J. 1999, ApJ, 519, 486

Miller Jr., R. G. 1981, Survival Analysis (John Wiley \& Sons)

Morton, D. C., Jian-sheng, C., Wright, A. E., Peterson, B. A., \& Jauncey, D. L. 1980, MNRAS, 193, 399

Nestor, D. B., Rao, S. M., Turnshek, D. A., et al. 2001, in Extragalactic Gas at Low Redshift, ed. J. S. Mulchaey, \& J. T. Stocke, ASP Conf. Ser., 254, 34

Payne, H. E., Salpeter, E. E., \& Terzian, Y. 1982, ApJS, 48, 199

Perlman, E. S., Stocke, J. T., Carilli, C. L., et al. 2002, AJ, 124, 2401

Petitjean, P., Théodore, B., Smette, A., \& Lespine, Y. 1996, A\&A, 313, L25

Petitjean, P., Srianand, R., \& Ledoux, C. 2000, A\&A, 364, L26

Pettini, M., Smith, L. J., Hunstead, R. W., \& King, D. L. 1994, ApJ, 426, 79

Pettini, M., King, D. L., Smith, L. J., \& Hunstead, R. W. 1997, ApJ, 478,536

Prochaska, J. X., \& Wolfe, A. M. 1997, ApJ, 487, 73

Prochaska, J. X., \& Wolfe, A. M. 1999, ApJS, 121, 369

Prochaska, J. X., Wolfe, A. M., Tytler, D. et al. 2001, ApJS, 137, 21

Rao, S. M., \& Turnshek, D. A. 1998, ApJ, 500, L115

Rao, S. M., \& Turnshek, D. A. 2000, ApJS, 130, 1

Roberts, M. S., Brown, R. L., Brundage, W. D., et al. 1976, AJ, 81, 293 
Rohlfs, K. 1986, Tools of Radio Astronomy (Berlin, Heidelberg: Springer-Verlag)

Saikia, D. J., Junor, W., Cornwell, T. J., Muxlow, T. W. B., \& Shastri, P. 1990, MNRAS, 245, 408

Schaye, J., ApJ, 559, L1

Spaans, M., \& Carollo, C. M. 1997, ApJ, 482, L3

Spaans, M., \& Norman, C. A. 1997, ApJ, 483, 87

Steidel, C. C., Pettini, M., Dickinson, M., \& Persson, S. E. 1994, ApJ, 437,75

Steidel, C. C., Bowen, D. V., Blades, J. C., \& Dickson, M. 1995, ApJ, 440, L45

Stocke, J. T., Wurtz, R., Wang, Q., Elston, R., \& Jannuzi, B. T. 1992, ApJ, 400, L17

Turnshek, D. A., \& Rao, S. M. 2002, ApJ, 572, L7

Vladilo, G., Centurión, M., Falomo, R., \& Molaro, P. 1997, A\&A, 327,47
White, R. L., \& Becker, R. H. 1992, ApJS, 79, 331

White, R. L., Kinney, A. L., \& Becker, R. H. 1993, ApJ, 407, 456

Wilkinson, P. N., Browne, I. W. A., Patnaik, A. R., Wrobel, J. M., \& Sorathia, B. 1998, MNRAS, 300, 790

Wolfe, A. M., \& Davis, M. M. 1979, AJ, 84, 699

Wolfe, A. M., Briggs, F. H., \& Jauncey, D. L. 1981, ApJ, 248, 460

Wolfe, A. M., Briggs, F. H., Turnshek, D. A., et al. 1985, ApJ, 294, L67

Wolfe, A. M., Turnshek, D. A., Smith, H. E., \& Cohen, R. D. 1986, ApJS, 61, 249

Wolfire, M. G., Hollenbach, D., McKee, C. F., Tielens, A. G. G. M., \& Bakes, E. L. O. 1995, ApJ, 443, 152

Wright, A., \& Otrupcek, R., ed. 1990, Parkes catalogue, Australia Telescope National Facility

Young, L. M., \& Lo, K. Y. 1997, ApJ, 490, 710 